\title{
Preparation and characterization of superporous hydrogel based on different polymers
}

\author{
Hitesh V Chavda, Rupal D Patel, Ishan P Modhia, Chhagan N Patel ${ }^{1}$ \\ Departments of Pharmaceutics and Pharmaceutical Technology, ${ }^{1}$ Pharmaceutical Chemistry, Shri Sarvajanik Pharmacy College, Gujarat \\ Technological University, Mehsana, Gujarat, India
}

\begin{abstract}
Introduction: Superporous hydrogel (SPH) swells very rapidly in a shorter period of time to an equilibrium size and contains highly porous structure. Aim: The synthesis of SPH of poly (acrylamide-co-acrylic acid) and its composites viz. Ac-Di-Sol and polyvinylpyrollidone (PVP) was carried out by solution polymerization. Materials and Methods: The SPH and SPH composites (SPHCs) were characterized by measurement of apparent density, porosity, swelling, mechanical strength, and scanning electron microscopy (SEM) studies. Results: FTIR studies confirmed the existence of acrylamide and acrylic acid in SPH. In distilled water SPH showed tremendous increase in equilibrium swelling capacity with conventional SPH as compared to its SPHCs of Ac-Di-Sol and PVP due to the increased in physical cross-linking network, respectively. The presence of Ac-Di-Sol and PVP in SPHCs increased the mechanical strength as compared to conventional SPH which is suitable for gastric retention. SEM pictures clearly indicated the formation of interconnected pores and capillary channels. Conclusion: The amount and type of polymers used affect almost all the characterization parameters of SPHs, and hence, depending upon the applications perspective such polymers could be used in drug delivery systems, successfully.
\end{abstract}

Key words: Ac-Di-Sol, mechanical strength, PVP, swelling

\section{INTRODUCTION}

Hydrogels are hydrophilic cross-linked polymers able to absorb large amounts of water and they are water insoluble. ${ }^{[1-3]}$ The swelling properties of hydrogels are mainly related to the network elasticity, the presence of hydrophilic functional groups, the cross-link density, and porosity. A variety of stimuli sensitive hydrogels have been studied, but in many cases, slow response to environmental stimuli limited their effective uses. ${ }^{[4]}$ Although such slow swelling is beneficial for many applications, there are many situations where a fast swelling polymer is more desirable. Therefore, a new generation of hydrogels, which swell and absorb water very rapidly, has been developed. Examples of

\section{Address for correspondence:}

Dr. Hitesh V. Chavda,

Department of Pharmaceutics and Pharmaceutical Technology, Shri

Sarvajanik Pharmacy College, Gujarat Technological University,

Near-Arvind Baug, Mehsana, Gujarat - 384001, India.

E-mail: hitcvd@yahoo.com

\begin{tabular}{|l|l|}
\hline \multicolumn{2}{|c|}{ Access this article online } \\
\hline Quick Response Code: & Website: \\
\hline & www.jpionline.org \\
\cline { 2 - 2 } & DOI: \\
\hline
\end{tabular}

this new generation are superporous hydrogels (SPHs), which swell to an equilibrium size in a short period of time. ${ }^{[5]}$

SPH, A new generation of hydrogel is the three-dimensional network of hydrophilic polymer with numerous pores larger than $100 \mu \mathrm{m}$ to $1 \mathrm{~mm}$ connected together to form open channel structures. SPHs, which absorb water very rapidly and swells to equilibrium size in a short period due to the presence of interconnected microscopic pores. ${ }^{[6]}$ The first approach for $\mathrm{SPH}$ synthesis involves cross-linking of co-monomers using multifunctional co-monomer, which acts as cross-linking agent. Polymerization reaction and Gas blowing techniques are mainly used to synthesize SPHs. Inorganic carbonates such as sodium bicarbonates are the commonly used foaming agents, which have been safely applied in drug delivery systems. The second method involves copolymerization of linear polymers by irradiation or by chemical compounds. ${ }^{[7]}$ Several important characteristic of SPHs includes their fast swelling, higher swelling ratio, and surface slipperiness, which make them an excellent candidate material to develop gastric retention devices. ${ }^{[8]}$ The first generation of SPHs are prepared from highly hydrophilic acrylamide, acrylic acid salts and sulfopropyl acrylate. These SPHs swell fast and due to the small fraction of the polymer in the swollen state, they are sometimes difficult to handle without breaking. ${ }^{[9]}$ $\mathrm{SPH}$ composites, as the second generation of SPHs, possess improved mechanical properties with composite agents such as Ac-Di-Sol and polyvinylpyrollidone (PVP) serving as the local 
point of physical entanglement of the formed polymer chains which have the function of improving the SPH strength. The mechanical properties of the SPHs are affected by formulation variables, such as the amount of cross-linking agent, the type of plasticizer, the type of monomer and the amount of blowing agent. Composite materials such as Ac-Di-Sol and PVP are the hydrophilic particulate materials which have been commonly used as disintegrants in pharmaceutical tablet. ${ }^{[10,11]}$ Acrylamide (AM) and Acrylic acid (AA) were chosen as the base monomers for their high water affinity and fast copolymerization velocity which are responsible for the high swelling capacity. ${ }^{[12,13]}$ The applications of SPHs and SPH composites (SPHCs) in the field of pharmaceutics involved preparation of gastric retention devices, peroral peptide delivery systems, fast-dissolving tablets, diet aid, occlusion devices and other commercial applications like preparation toy products for children. A composite material is used in SPHCs, is a cross-linked hydrophilic polymer that can absorb the reaction mixture of monomers, cross-linker, initiator and other components of the SPH preparation. During polymerization, the composite material serves as the local point of physical entanglement of the formed polymer chains. ${ }^{[14]}$

The objective for the current investigation was to synthesis $\mathrm{SPH}$ and SPHCs containing Ac-Di-Sol and PVP as a composite material to improve the characteristics of conventional SPHs and comparison thereof.

\section{MATERIALS AND METHODS}

AM was obtained from burgoyne burbidges and Co. Pvt. Ltd., Mumbai, India. AA, PVP, Ac-Di-Sol, N,N'methylenebisacrylamide (BIS), Span 80, ammonium persulphate (APS), N,N,N',N'-tetramethylethylenediamine (TEMED), and $\mathrm{NaHCO}_{3}$ were purchased from SD Fine Chem. Ltd., Mumbai, India. Distilled water was prepared in laboratory. Used all other chemicals were of analytical grade and with quality.

\section{SPH and SPHCs synthesis}

All the ingredients from 1 to 8 as shown in Table 1 were added subsequently into a test tube at room temperature and $\mathrm{pH} 5.0$ was adjusted with $5 \mathrm{M} \mathrm{NaOH}$ solution. After that APS was added to the reaction mixture. Polymerization was allowed to continue for ten minutes and the mixture was shaken vigorously. Finally, $\mathrm{NaHCO}_{3}$ was added quickly to the solution. Prepared SPHs were dehydrated using hexane. Thereafter, the SPHs were removed with the forceps and put in hot air oven at $60^{\circ} \mathrm{C}$ for 24 hours for complete removal of solvent, to ensure complete drying of SPHs and stored in airtight container until the further use.

\section{Scanning electron microscopy studies}

The texture of hydrogel was analyzed by scanning electron microscopy (SEM) to ensure that hydrogel retains its structure. The dried SPH and SPHCs were cut to expose their inner structure and used for SEM studies. The morphology and porous structure of the SPHC was examined using SEM JSM-5600 scanning electron microscope (JEOL worldwide, India), with an operating voltage of $10 \mathrm{kV}$.

\section{Density measurements}

Density measurement was carried out using the solvent displacement method. Dried SPH were used for the density measurement, which actually show the apparent densities. Pieces of SPH were taken and weighed in order to determine the mass of each piece. A piece of polymer was immersed in a measured volume of hexane, and the increase in the hexane volume was measured. The density was calculated from the equation 1 .

Density $=\mathrm{M}_{\mathrm{SPH}} / \mathrm{V}_{\mathrm{SPH}}$

Where, $\mathrm{V}_{\mathrm{SPH}}$ is the volume of solvent displaced by SPH and $\mathrm{M}_{\mathrm{SPH}}$ is the mass of dried SPH.

\section{Swelling studies}

The dried SPH were used to determine their equilibrium swelling ratio in distilled water. The equilibrium swelling ratio can be calculated from the following equation 2 .

$\mathrm{Q}=\left(\mathrm{M}_{\mathrm{s}}-\mathrm{M}_{\mathrm{d}}\right) / \mathrm{M}_{\mathrm{d}}$

Where, $\mathrm{Q}$ is the equilibrium swelling ratio, $\mathrm{M}_{\mathrm{s}}$ and $\mathrm{M}_{\mathrm{d}}$ are the masses in swollen state and dried state, respectively. Initially, $\mathrm{M}_{\mathrm{d}}$ of a piece of polymer was measured by weight in dried state and then it was immersed in an excess of distilled water for swelling. The swollen SPH were put on a grid boat with a mesh size of $1 \mathrm{~mm}$. This method permits simple handling of

Table 1: Composition of SPHs and SPHCs

\begin{tabular}{|c|c|c|c|c|c|c|c|c|}
\hline Ingredients & $\mathrm{B}_{1}$ & $\mathrm{~B}_{2}$ & $\mathrm{~B}_{3}$ & $\mathrm{~B}_{4}$ & $B_{5}$ & $\mathrm{~B}_{6}$ & $\mathrm{~B}_{7}$ & $\mathrm{~B}_{8}$ \\
\hline $\mathrm{AM}(50 \% \mathrm{w} / \mathrm{v})$ & $600 \mu \mathrm{l}$ & $300 \mu \mathrm{l}$ & $300 \mu \mathrm{l}$ & $300 \mu \mathrm{l}$ & $300 \mu \mathrm{l}$ & $300 \mu \mathrm{l}$ & $300 \mu \mathrm{l}$ & $300 \mu \mathrm{l}$ \\
\hline $\mathrm{AA}(50 \% \mathrm{v} / \mathrm{v})$ & $400 \mu \mathrm{l}$ & $200 \mu \mathrm{l}$ & $200 \mu \mathrm{l}$ & $200 \mu \mathrm{l}$ & $200 \mu \mathrm{l}$ & $200 \mu \mathrm{l}$ & $200 \mu \mathrm{l}$ & $200 \mu \mathrm{l}$ \\
\hline $\mathrm{BIS}(2.5 \% \mathrm{w} / \mathrm{v})$ & $200 \mu \mathrm{l}$ & $100 \mu \mathrm{l}$ & $100 \mu \mathrm{l}$ & $100 \mu \mathrm{l}$ & $100 \mu \mathrm{l}$ & $100 \mu \mathrm{l}$ & $100 \mu \mathrm{l}$ & $100 \mu \mathrm{l}$ \\
\hline Span $80(10 \% \mathrm{v} / \mathrm{v})$ & $30 \mu \mathrm{l}$ & $30 \mu \mathrm{l}$ & $30 \mu \mathrm{l}$ & $30 \mu \mathrm{l}$ & $30 \mu \mathrm{l}$ & $30 \mu \mathrm{l}$ & $30 \mu \mathrm{l}$ & $30 \mu \mathrm{l}$ \\
\hline Ac-Di-Sol & - & - & $50 \mathrm{mg}$ & - & $150 \mathrm{mg}$ & - & $250 \mathrm{mg}$ & - \\
\hline PVP & - & - & - & $50 \mathrm{mg}$ & - & $150 \mathrm{mg}$ & - & $250 \mathrm{mg}$ \\
\hline TEMED $(20 \% \mathrm{v} / \mathrm{v})$ & $20 \mu \mathrm{l}$ & $20 \mu \mathrm{l}$ & $20 \mu \mathrm{l}$ & $20 \mu \mathrm{l}$ & $20 \mu \mathrm{l}$ & $20 \mu \mathrm{l}$ & $20 \mu \mathrm{l}$ & $20 \mu \mathrm{l}$ \\
\hline Distilled water & $330 \mu \mathrm{l}$ & $330 \mu \mathrm{l}$ & $330 \mu \mathrm{l}$ & $330 \mu \mathrm{l}$ & $330 \mu \mathrm{l}$ & $330 \mu \mathrm{l}$ & $330 \mu \mathrm{l}$ & $330 \mu \mathrm{l}$ \\
\hline APS $(20 \% \mathrm{w} / \mathrm{v})$ & $45 \mu \mathrm{l}$ & $45 \mu \mathrm{l}$ & $45 \mu \mathrm{l}$ & $45 \mu \mathrm{l}$ & $45 \mu \mathrm{l}$ & $45 \mu \mathrm{l}$ & $45 \mu \mathrm{l}$ & $45 \mu \mathrm{l}$ \\
\hline $\mathrm{NaHCO}_{3}$ & $100 \mathrm{mg}$ & $200 \mathrm{mg}$ & $200 \mathrm{mg}$ & $200 \mathrm{mg}$ & $200 \mathrm{mg}$ & $200 \mathrm{mg}$ & $200 \mathrm{mg}$ & $200 \mathrm{mg}$ \\
\hline
\end{tabular}

AM: Acrylamide, AA: Acrylic acid, BIS: N,N'-methylenebisacrylamide, APS: Ammonium persulphate, TEMED: N,N, $N^{\prime}, N^{\prime}$-tetramethylethylenediamine, PVP: Polyvinylpyrollidone 
SPH and SPHCs in water while not altering the mechanical integrity. Using the grid boat polymers were removed from water; dried gently by tissue paper so as to get rid of adhering water. At specific time intervals the polymer was removed from the water and weighed in order to measure $\mathrm{M}_{s} \cdot{ }^{[5]}$ When the weight became constant it was considered as $\mathrm{M}_{\mathrm{s}}$ and the time was considered as swelling time.

\section{Porosity measurements}

For porosity measurement, dried SPH were immersed in hexane over night and weighed when excess solvent on the surface was blotted. The porosity was calculated from equation 3 .

$$
\text { Porosity }=\mathrm{V}_{\mathrm{P}} / \mathrm{V}_{\mathrm{T}}
$$

Where $\mathrm{V}_{\mathrm{T}}$ is the $\mathrm{SPH}$ total volume and $\mathrm{V}_{\mathrm{P}}$ is the $\mathrm{SPH}$ pore volume. SPH total volume could be measured from its dimensions (cylindrical in shape). Pore volume could be determined by subtracting the $\mathrm{V}_{\mathrm{SPH}}$ from $\mathrm{V}_{\mathrm{T}}$.

\section{Mechanical strength studies}

The penetration pressure (PP) of the SPHCs was measured employing a bench comparator as described by Chen et al. with modifications. ${ }^{[10]}$ The absolutely swollen hydrogel was place longitudinally beneath the lower bit and weights were successively applied to the higher bit till the polymer fully fractured. The ultimate compressive force was browsed from the gauge and the penetration pressure ${ }^{[15]}$ was calculated from the following equation 4 .

$$
\mathrm{PP}=\mathrm{F}_{\mathrm{u}} / \mathrm{S}
$$

Where, $\mathrm{F}_{\mathrm{u}}$ is the ultimate compressive force at complete breakage of the SPH and SPHCs and S is the area of the lower touch.

\section{FT-IR studies}

The SPHs were dried and converted in powdered form for FT-IR study. The powdered samples were mixed with $\mathrm{KBr}$ and FT-IR spectrum was recorded over the range $600-4000 \mathrm{~cm}^{-1}$ using FTIR8400S Spectrometer (Shimadzu, Japan).

\section{RESULTS AND DISCUSSION}

\section{SPH and SPHCs synthesis}

In this procedure of $\mathrm{SPH}$ synthesis, $\mathrm{AA}$ and $\mathrm{AM}$ are the monomers. Ac-Di-Sol and PVP have the function of improving the SPH strength. BIS and span 80 were used as cross linker and foam stabilizer, respectively. Foam was generated by carbon dioxide due the reaction between acid and sodium bicarbonate. Homogeneous SPH with many pores could be obtained only when the polymerization and foam stabilization occurred simultaneously. As shown in Table 2, SPH was not formed which showed that variables in the amount of monomer and cross-linker depends on formation of SPHs. Span 80 was very important as a surfactant to create porous structure.APS was used as a polymerization initiator and TEMED as a catalyst. One of the important factors that influence the synthesis of SPH was the $\mathrm{pH}$ of the $\mathrm{AA}$ monomer solution. To make SPH with interconnected and uniform pores the monomer solution must have good foaming and foam stabilizing mechanism. At the $\mathrm{pH}$ 5.0, SPH with well-distributed pores were produced because of the stability and the proper formation rate of the foam. Another role of Ac-Di-Sol and PVP is to stabilize the foam during the synthesis and increases the physical cross-linking density of SPH. Prepared SPH and SPHCs are shown in Figure 1. Gastric retention devices utilizing SPHCs are reported which includes

\begin{tabular}{|c|c|c|c|c|c|}
\hline Batch & Density (g/cc) & Porosity (\%) & Swelling ratio (\%) & Swelling time (min) & Penetration pressure $\left(\mathrm{g} / \mathrm{cm}^{2}\right)$ \\
\hline $\mathrm{B} 1^{*}$ & - & - & - & - & - \\
\hline B2 & $0.730 \pm 0.04$ & $70.40 \pm 0.30$ & $89.70 \pm 7.50$ & $02 \pm 0.37$ & $11 \pm 2$ \\
\hline B3 & $0.704 \pm 0.14$ & $72.35 \pm 0.19$ & $41.57 \pm 9.42$ & $04 \pm 1.24$ & $24 \pm 9$ \\
\hline B4 & $0.695 \pm 0.57$ & $76.24 \pm 0.23$ & $58.70 \pm 4.52$ & $05 \pm 1.62$ & $39 \pm 5$ \\
\hline B5 & $0.627 \pm 0.02$ & $89.87 \pm 0.09$ & $62.49 \pm 3.50$ & $11 \pm 2.16$ & $264 \pm 32$ \\
\hline B6 & $0.556 \pm 0.70$ & $90.29 \pm 0.07$ & $49.16 \pm 2.34$ & $32 \pm 4.81$ & $872 \pm 26$ \\
\hline B7 & $0.427 \pm 0.21$ & $62.54 \pm 0.16$ & $12.18 \pm 4.60$ & $11 \pm 3.05$ & $270 \pm 42$ \\
\hline B8 & $0.405 \pm 0.09$ & $69.18 \pm 0.11$ & $9.46 \pm 2.41$ & $15 \pm 2.48$ & $378 \pm 18$ \\
\hline
\end{tabular}
drug and polymer mixture as a core loaded in SPHC conveyer

Mean $\pm S D, n=3$ * SPH was not formed
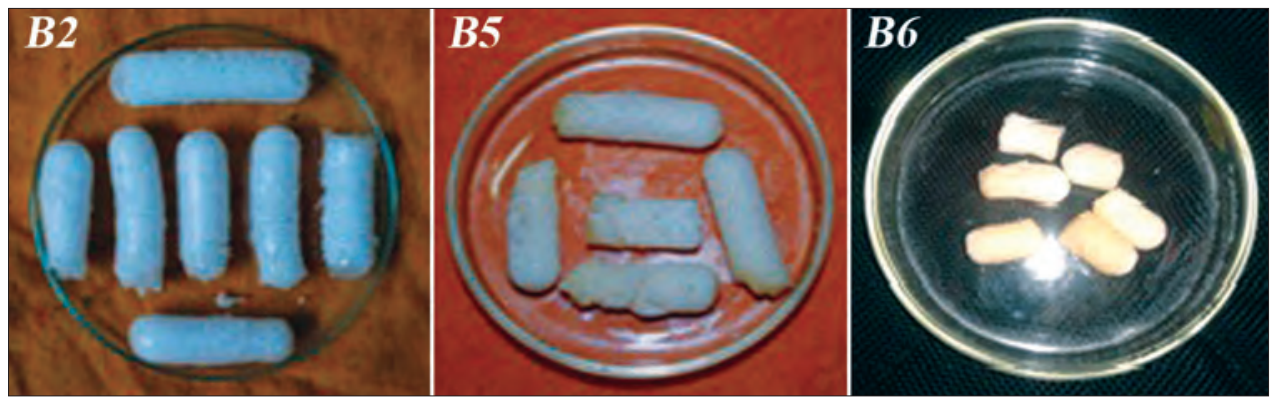

Figure 1: Photograph of petridishes (inner diameter=7.5 cm) containing dried SPH (B2) and SPHCs (B5, B6) 
systems filled in capsules for peptide and other drug delivery systems. ${ }^{[14,16]}$ Dorkoosh et al. reported that the retention of SPHC polymer in human volunteers shown no discomfort. ${ }^{[17]}$ Risbud and Bhonde showed that polyacrylamide-Chitosan hydrogel were in vitro biocompatible as they showed no cytotoxic effects on NIH3T3 and HeLa cells determined by MTT and neutral red assay. ${ }^{[18]}$ Yin et al. reported cytotoxicity assays of SPH on RBL-2H3 and Caco-2 cells caused minimal damage towards cell viability, lysosomal activity, and metabolic activity. ${ }^{[19]}$ These all indicates that SPH and SPHCs are suitable and safe for drug delivery systems.

\section{SEM studies}

Figure 2 shows the SEM images of SPH of poly (AM-co-AA), poly (AM-co-AA) with Ac-Di-Sol and PVP. SPH and SPHCs with uniform superporous structure and interconnected pores were produced when the monomer solution have good foaming and foam stabilizing mechanism. As observed in SEM images the structure of SPH contained numerous pores interconnected to each other. The capillary channels are clearly observed that enable water molecules to enter into the SPH networks. SPH of poly (AM-co-AA) shows more numbers of small size pores compared to SPHCs. SPHC with Ac-Di-Sol shows numerous small size pores, out of which many pores observed to be blocked due to the presence of Ac-Di-Sol. SPHC with PVP shows quite less numbers of interconnected pores and the porous structure is blocked at many points which reflects that PVP as a composite material increases the crosslink density of SPH and so the mechanical strength.

\section{Density measurements}

As shown in Table 2, the density of the synthesized SPHs was obtained between 0.4 to $0.8 \mathrm{~g} / \mathrm{cc}$. The density of SPHs was lower than compared with the conventional hydrogels, as SPHs possess lots of pores. Ac-Di-Sol and PVP particles were interlocked with the SPH matrix to form an integral unit. As shown in Table 2, the rigid Ac-Di-Sol and PVP particles maintained the porous structure with $150 \mathrm{mg}$ and so that the SPH shrank much less and showed lower density as compared to poly (AM-co-AA).

\section{Swelling studies}

The important characteristic of SPH is its fast swelling ability. Swelling parameters of different SPHs in distilled water are shown in Table 2. Poly (AM-co-AA) SPH showed much higher swelling ratio and less equilibrium swelling time owing to their highly porous structures compared to that of SPHCs of Ac-DiSol and PVP. Amount of Ac-Di-Sol and PVP resulted in decrease in density and swelling ratio respectively. Amount of Ac-Di-Sol and PVP of $200 \mathrm{mg}$ showed very less swelling ratio which was not in the characteristic of SPHs. The slower swelling time may be due to the partial blockage of capillary channel with fibers of Ac-Di-Sol and PVP. Since, Ac-Di-Sol and PVP are not expected to participate as a chemical cross-linking agent, but it was act as a physical cross-linking agent. As shown in Table 2, as the

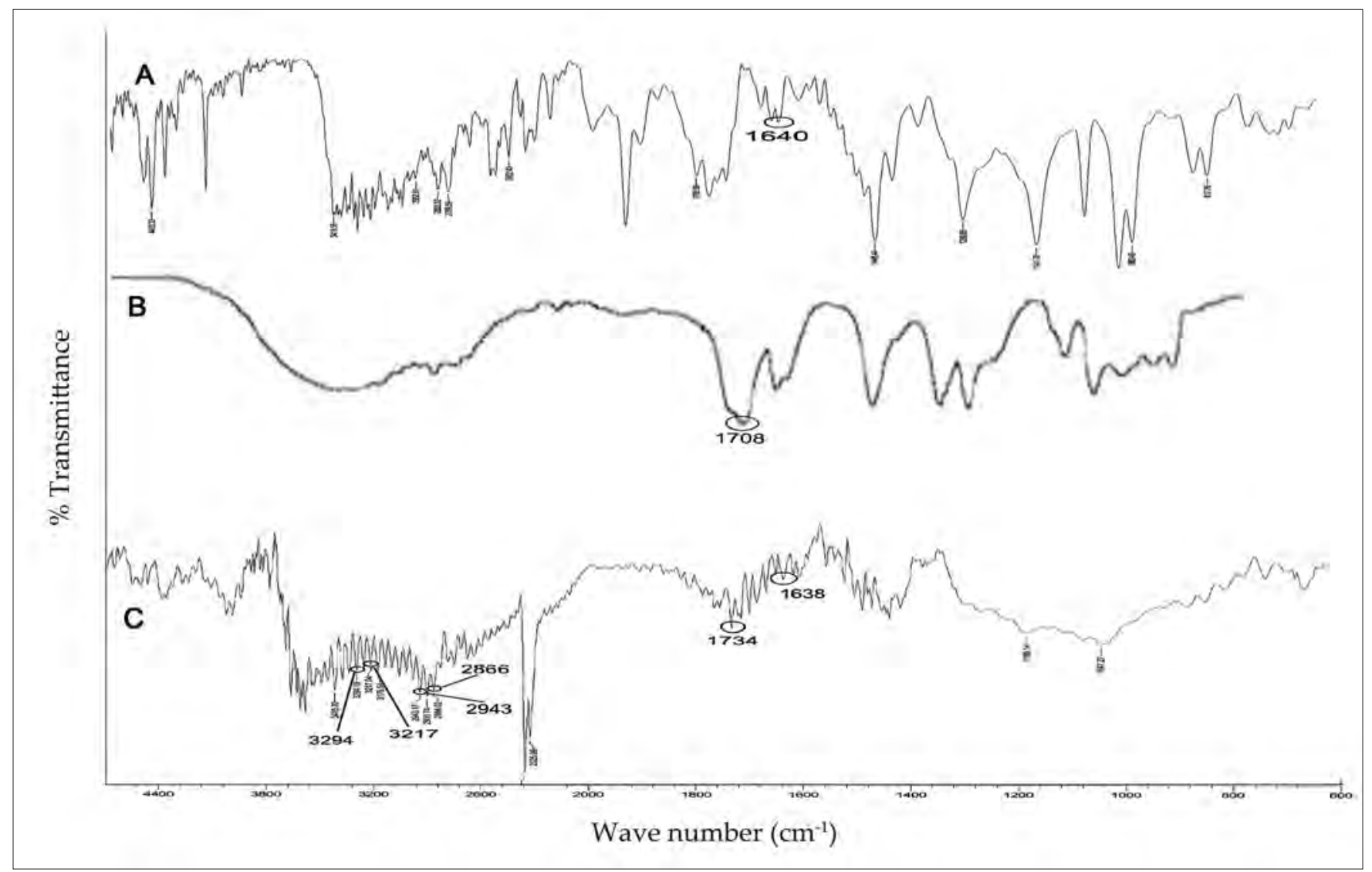

Figure 2: FTIR spectra of $A M, A A^{[21]}$ and $S P H$ of poly (AM-co-AA) 

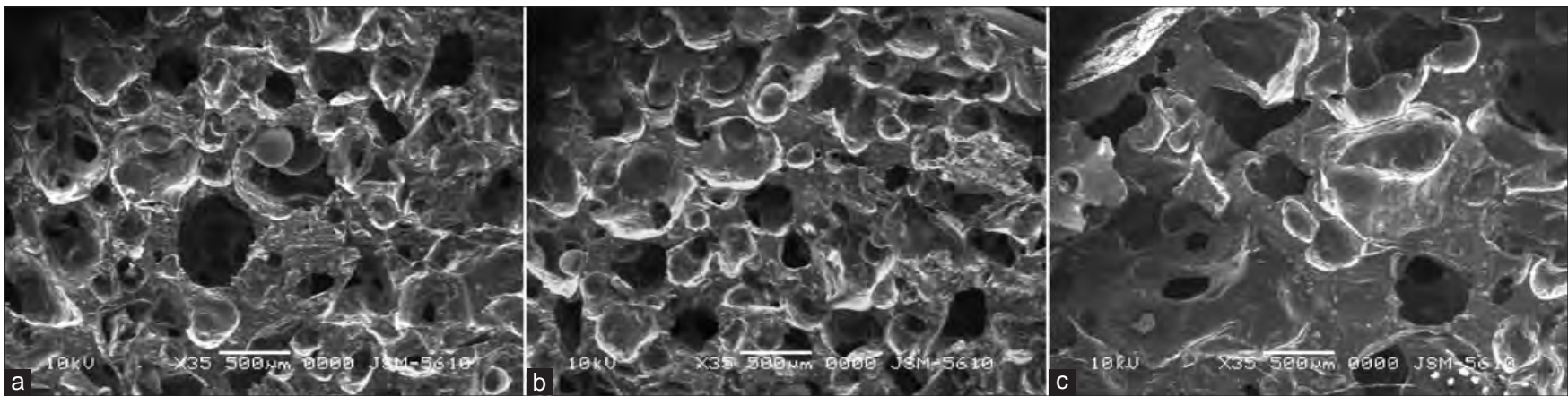

Figure 3: SEM pictures of SPH and SPHCs: (a) SPH of poly (AM-co-AA); (b) SPHC of poly (AM-co-AA) with Ac-Di-Sol; (c) SPHC of poly (AMco-AA) with PVP

amount of Ac-Di-Sol and PVP increased, the swelling ratio of SPHs was decreased. When Ac-Di-Sol and PVP were mixed with the monomer solution, they swelled so that monomers and cross-linker were absorbed into their network structure. During the synthesis of SPH, the absorbed monomer and BIS and along with those that were not absorbed, all the participated in polymerization process further leading to the formation of interpenetration polymer network (IPN) and this IPN formation was limited to the Ac-Di-Sol and PVP provided additional cross-linking. Further decrease in swelling ratios of SPH with Ac-Di-Sol and PVP incorporated SPH was partially due to the increase in physical cross-linking density, respectively.

\section{Porosity measurements}

The porosity data are shown in Table 2. The porosity was found to be independent of composite materials used, which might be due to the blockage of some capillaries and so solvent could not penetrated well. At higher concentration of composite materials porosity was decreased due to the increase crosslink density.

\section{Mechanical strength studies}

One of most salient property for a gastric retention SPH is its structural integrity network. A SPH should be able to withstand the pressure expected in the stomach during repeated gastric contractions. Formulation variables viz. amount of cross-linker, type of monomer, amount of blowing agent, amount of Ac-Di-Sol and PVP, process variables affect the mechanical strength of the SPHs. The maximum pressure during the gastric contraction was ranges from $50-70 \mathrm{~cm}$ water. ${ }^{[20]}$ Penetration pressures of prepared SPHs are shown in Table 2, as the amount of Ac-Di-Sol and PVP increases, the mechanical strength of SPHs increases. In this study, the effect of the presence of the Ac-Di-Sol and PVP decreases the physical cross-link density of the SPH which was the possible reason for the improved structural integrity.

\section{FT-IR studies}

Figure 3 shows the FT-IR spectra of AM, AA, and synthesized $\mathrm{SPH}$, respectively. AM and AA showed characteristic carbonyl stretching at $1640 \mathrm{~cm}^{-1}$ and $1708 \mathrm{~cm}^{-1}$ along with C-H stretching above $3000 \mathrm{~cm}^{-1} .^{[21]}$ Peaks at $1638 \mathrm{~cm}^{-1}$ and $1734 \mathrm{~cm}^{-1}$ signified the presence of amide and carboxylic groups, which confirmed the existence of $\mathrm{P}$ (AA-co-AM) in the SPH. This was further confirmed by the peaks at 3294 and $3217 \mathrm{~cm}^{-1}$ due to amide- $\mathrm{NH}_{2}$ stretching along with the shift of olefinic $\mathrm{C}-\mathrm{H}$ stretching above $3000 \mathrm{~cm}^{-1}$ to below $3000 \mathrm{~cm}^{-1}$ (2943 and $2806 \mathrm{~cm}^{-1}$ ). Locations of the peaks slightly changed, which was attributed to the crosslinking.

\section{CONCLUSIONS}

SPH and their composites possessed porous structure and were less dense compared to GI fluid. The mechanical stability and porosity of poly (AM-co-AA) SPH were less compared to their composites, Ac-Di-Sol and PVP. The improved mechanical strength of SPHCs prevents the breakage of hydrogel during gastric contraction. The equilibrium swelling ratio and swelling time reflects the extended swelling capacity and rapid swelling behaviour of prepared hydrogels. The amount of cross-linker, monomer, Ac-Di-Sol and PVP affect the characterization parameters of the SPHs. Prepared SPHCs of Ac-Di-Sol and PVP resulted in improving structural integrity.

\section{REFERENCES}

1. Guzman J, Iglesias M, Riande E, Compaii V, Andrio A. Synthesis and polymerization of acrylic monomers with hydrophilic long side groups. Oxygen transport through water swollen membranes prepared from these polymers. Polymer 1997;38:5227-32.

2. Sen M, Guven O. Prediction of swelling behaviour of hydrogels containing diprotic acid moieties. Polymer 1998;39:1165-72.

3. English AE, Tanaka T, Edelman ER. Polymer and solution ion shielding in polyampholytic hydrogels. Polymer 1998;39:5893-7.

4. Allan SH. Hydrogels for biomedical applications. Adv Drug Deliver Rev 2002;43:3-12.

5. Chen J, Park H, Park K. Synthesis of superporous hydrogels: Hydrogels with fast swelling and superabsorbent properties. J Biomed Mater Res 1999;44:53-62.

6. Park K. Superporous hydrogels for pharmaceutical and other applications. Drug Deliver Technol 2002;38:40-4.

7. Satish CS, Satish KP, Shivakumar HG. Hydrogels as controlled drug delivery systems: Synthesis, cross-linking, water and drug transport mechanism. Indian J Pharm Sci 2006;68:133-40.

8. Qiu Y, Park K. Superporous IPN hydrogels having enhanced mechanical properties. AAPS PharmSciTech 2003;4:E51. 
9. Omidian H, Rocca JG, Park K. Advances in superporous hydrogels. J Control Release 2005;102:3-12.

10. Chen J, Park K. Synthesis and characterization of superporous hydrogel composites. J Control Release 2000;65:73-82.

11. Polnok A, Verhoef JC, Borchard G, Sarisuta N, Junginger HE. In vitro evaluation of intestinal absorption of desmopressin using drug-delivery systems based on superporous hydrogels. Int $\mathrm{J}$ Pharm 2004;269:303-10.

12. Park $\mathrm{K}$, Chen J, Park $\mathrm{H}$. Hydrogel composites and superporous hydrogel composites having fast swelling, high mechanical strength and superabsorbent properties. US Patent No. 6271278; 2001.

13. Chavda HV, Patel CN. Preparation and characterization of swellable polymer-based superporous hydrogel composite of poly (acrylamide-co-acrylic acid). Trends Biomater Artif Organs 2010;24:83-9.

14. Park K. Superporous hydrogels for pharmaceutical and other applications. Drug Del Tech [serial on the internet]. 2002;2:7. Available from: http://www.drugdeliverytech.com/ ME2/dirmod.asp?sid $=$ and $\mathrm{nm}=$ and type=Publishing and mod=Publications $\% 3 A \% 3 A A r t i c l e ~ a n d ~ m i d=8 F 3 A 70274218419$ 78F18BE895F87F791 and tier=4 and id=B86C8DCD26724945 B6CC7624176F1F33. [cited 2012 Jul 27].

15. Tang $\mathrm{C}$, Yin $\mathrm{CH}$, Pei YY, Zhang M, Wu LF. New superporous hydrogels composites based on aqueous Carbopol solution (SPHCcs): Synthesis, characterization and in vitro bioadhesive force studies. Eur Polym J. 2005;41:557-62.
16. Chavda HV, Patel CN. Preparation and In Vitro Evaluation of a Stomach Specific Drug Delivery System based on Superporous Hydrogel Composite. Indian J Pharm Sci 2011;73:30-7.

17. Dorkoosh FA, Stokkel MP, Blok D, Borchard G, Rafiee-Tehrani $\mathrm{M}$, Verhoef $\mathrm{JC}$, et al. Feasibility study on the retention of superporous hydrogel composite polymer in the intestinal tract of man using scintigraphy. J Control Release 2004;99:199-206.

18. Risbud MV, Bhonde RR. Polyacrylamide-chitosan hydrogels: In vitro biocompatibility and sustained antibiotic release studies. Drug Deliv 2000;7:69-75.

19. Yin L, Zhao X, Cui L, Ding J, He M, Tang C, et al. Cytotoxicity and genotoxicity of superporous hydrogel containing interpenetrating polymer networks. Food Chem Toxicol 2009;47:1139-45.

20. Chen J, Park H, Park K. Superporous hydrogel as a platform for oral controlled drug delivery. In: Wise DL, editor. Handbook of Pharmaceutical Controlled Release Technology, $2^{\text {nd }}$ edition. New York: Marcel Dekker, Inc.;2005. p. 211-24.

21. Yin L, Fei L, Cui F, Tang C, Yin C. Superporous hydrogels containing poly(acrylic acid-co-acrylamide)/O-carboxymethyl chitosan interpenetrating polymer networks. Biomaterials 2007;28:1258-66.

How to cite this article: Chavda HV, Patel RD, Modhia IP, Patel $\mathrm{CN}$. Preparation and characterization of superporous hydrogel based on different polymers. Int J Pharma Investig 2012;2:134-9. Source of Support: Nil. Conflict of Interest: None declared. 